\title{
Hybrid optical waveguide devices based on polymers and silica
}

\author{
Mart B.J. Diemeer* \\ University of Twente, Lightwave Devices Group, \\ P.O. Box 217, 7500 AE Enschede, The Netherlands
}

\begin{abstract}
The hybrid integration of polymer and silica in optical waveguides can yield devices that combine the excellent thermo-optic properties of polymers and the superior passive waveguiding properties of silica. The large difference and opposite sign of the thermo-optic coefficients of both classes of materials can be utilized to create athermal waveguide devices. In addition, it can be utilized in thermo-optic devices to induce local changes in the refractive index with boundaries that are sharply defined by the material interfaces and not by gradual thermal profiles. This can also yield devices with attractive thermo-optic behavior.
\end{abstract}

Keywords: hybrid integration, polymers, silica, optical waveguides, thermo-optic effect, athermal

\section{INTRODUCTION}

Both polymers and silica are used as waveguide materials in integrated optical devices. Polymers are mainly applied in thermo-optic devices because of their excellent thermo-optic properties. ${ }^{1}$ Their thermo-optic coefficient $\mathrm{dn}_{\text {polymer }} / \mathrm{dT}$ is about $-10^{-4}{ }^{\circ} \mathrm{C}^{-1}$ in glassy polymers and up to $-5.10^{-4}{ }^{\circ} \mathrm{C}^{-1}$ in rubbery polymers, while their thermal conductivity $\mathrm{T}_{\mathrm{c}}$ is as low as $0.2 \mathrm{Wm}^{-1} \mathrm{~K}^{-1}$. The propagation loss in fiber-compatible polymer waveguides @ $1.55 \mu \mathrm{m}$ ranks between 0.1 and $0.5 \mathrm{~dB} / \mathrm{cm}$.

Silica is widely used in passive, silica-on-silicon devices, like arrayed waveguide gratings (AWG's) and splitters, because it provides very low optical loss waveguides. ${ }^{2}$ The best silica waveguides have loss rates of $0.01 \mathrm{~dB} / \mathrm{cm}$. However the thermo-optic coefficient of silica, $\mathrm{dn}_{\text {silica }} / \mathrm{dT}$, of $+10^{-5}{ }^{\circ} \mathrm{C}^{-1}$ is more than an order of magnitude smaller than that of polymers, while its thermal conductivity of $1.4 \mathrm{Wm}^{-1} \mathrm{~K}^{-1}$ is 7 times larger than that of polymers. The required activation power in thermo-optic devices is inversely proportional to the thermo-optic coefficient of the waveguide and proportional to its thermal conductivity. Therefore silica devices require two orders of magnitude more power for activation than polymer devices.

The hybrid integration of polymer waveguides for thermo-optic activation and silica waveguides for passive functions is an approach where the best properties of both material systems are utilized to realize devices with enhanced performance. Polymers provide attractive properties for this approach, because high quality polymer layers can be deposited by simple, low temperature spincoating techniques onto silica waveguide structures. In addition polymers can be designed to yield good planarization, gap filling and adhesive properties. Moreover, the refractive index of polymers can be finely tuned in a broad range to make it compatible to silica waveguides.

Additional properties that can be utilized in the hybrid integration of polymers and silica in order to realize attractive devices are the difference in magnitude and sign of the thermo-optic coefficients. They can be used to make channel waveguides with an athermal effective index by applying a polymer cladding onto silica cores.

Another way to athermalize AWG's and other interferometric devices, based on the large difference in thermo-optic coefficients of polymers and silica, is to make optical path lengths or optical path length differences athermal by inserting small polymer segments in the core of silica waveguides.

The difference in thermo-optic coefficient can be also be utilized to induce regions with different refractive indices. These regions have boundaries that are sharply defined by the material boundaries and not by a gradual thermal profile. This can also yield efficient thermo-optic switching effects.

This paper presents a few examples of attractive hybrid polymer/silica waveguide devices.

* m.b.j.diemeer@el.utwente.nl; phone+31534895417; fax +31534893343; www.el.utwente.nl/tdm/ldg 


\section{POLYMER FILLED GAPS IN SILICA WAVEGUIDES}

The thermo-optic coefficient of rubbery polymers is so large that in some applications a waveguide section of less than $50 \mu \mathrm{m}$ is sufficient to create the required phase change. In fiber-compatible waveguides, a non-waveguiding gap of that size can be bridged without excessive loss due to diffraction. A simple to realize, polymer filled hole in silica channel waveguides can then be applied. Fig. 1 shows an example of such an approach (by NTT) applied in an AWG's in order to make it athermal. ${ }^{3}$

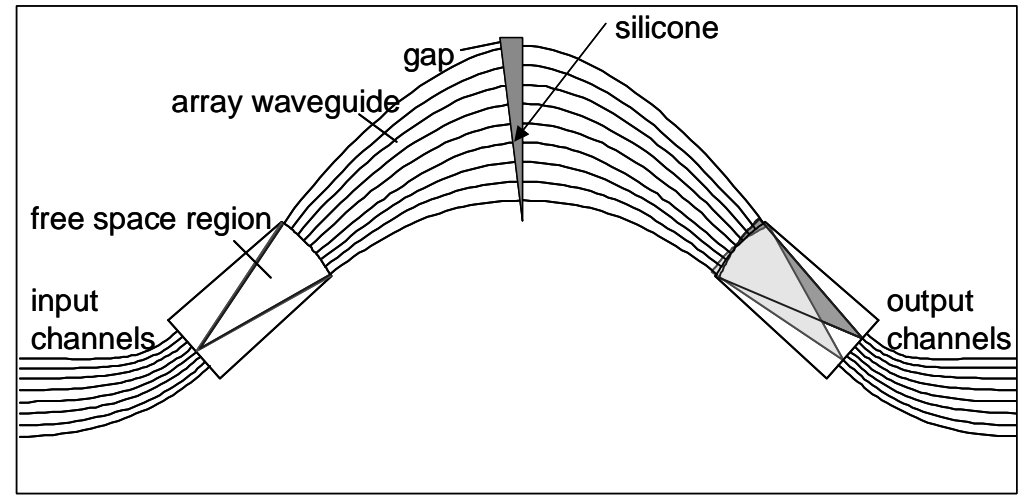

Fig. 1: Athermal AWG using a polymer filled gap

The triangular groove is designed such that the thermal change of the optical path differences in the silica waveguide channels is compensated by that of silicone filled gaps in these channels. The much larger (by a factor of 40) thermooptic coefficient of silicone compared to silica keeps the gap width at acceptable values for low excess loss. An excess loss of $2 \mathrm{~dB}$ was reported. The gap loss $\mathrm{G}_{\mathrm{L}}$ in $\mathrm{dB}$ 's is described by: ${ }^{4}$

$G_{L}=10 \cdot \log \left[1+\left(\frac{\lambda \cdot g}{2 \pi \cdot n \cdot r^{2}}\right)^{2}\right]$

where $\lambda$ is the wavelength, $\mathrm{g}$ is the gap, $\mathrm{r}$ is the spotsize radius of the mode and $\mathrm{n}$ is the refractive index of the polymer in the gap. The loss @ $1.55 \mu \mathrm{m}$ in a gap of $50 \mu \mathrm{m}$ filled with a polymer having a refractive index of 1.45 for channels with $\mathrm{r}=5 \mu \mathrm{m}$ is less than $0.5 \mathrm{~dB}$. The nonlinear dependence of $\mathrm{G}_{\mathrm{L}}$ on the gap size suggests segmentation of the gap in smaller gaps to reduce the loss. Five cascaded gaps of 10 microns yield a loss of only $0.1 \mathrm{~dB}$.

This segmentation approach has been applied by NTT to reduce the gap loss in their athermal AWG. ${ }^{5}$ Less than 0.4 $\mathrm{dB}$ was reported for a 16 channel $100 \mathrm{GHz}$ spaced AWG.

Mach Zehnder interferometric switches or attenuators are excellent devices for the gap filling approach. To illustrate this, the required phase shift of $\pi$ is calculated using an active elastomeric waveguide section. The thermo-optic coefficient for this material $\mathrm{dn}_{\text {polymer }} / \mathrm{dT}=-5.10^{-4}{ }^{\circ} \mathrm{C}^{-1}$. Assuming a moderate temperature rise of say 30 degrees and a wavelength of $\lambda=1.55 \mu \mathrm{m}$ and an active section length $\mathrm{G}$ in $\mu \mathrm{m}$, the phaseshift $\Delta \phi$ will be:

$\Delta \phi=\frac{2 \pi}{\lambda} \Delta n \cdot G=-2 \times 10^{-2} \pi \cdot G$

The required length of the active section is therefore only $50 \mu \mathrm{m}$ and the simple gap filling procedure would already give good results in a Mach Zehnder interferometer. A schematic picture of such a device is shown in fig.1. After the fabrication of the silica-on silicon device, holes are etched into the channel. These holes are backfilled with a liquid monomer that is UV or thermally cured to form the polymer. The heaters are defined onto the silica. They are meandering around the gaps for quick and efficient heating of the polymer. 


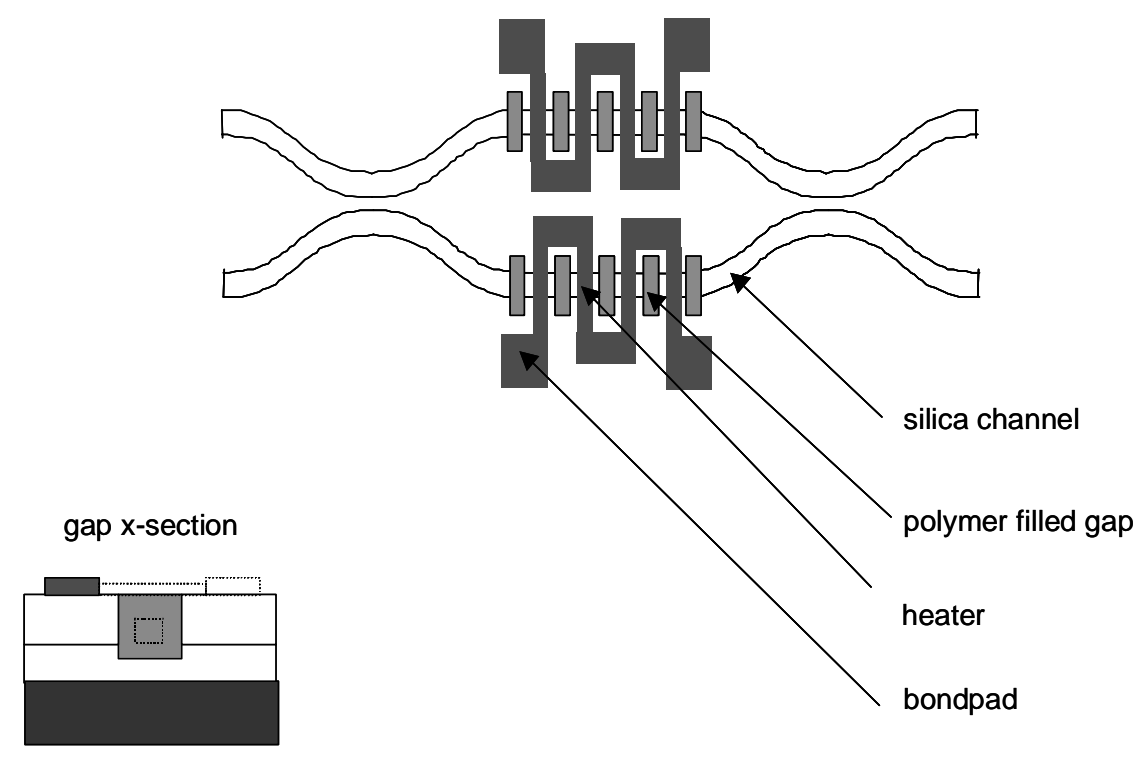

Fig. 2: Hybrid Mach Zehnder interferometer using polymer filled gaps.

The required power dissipation $\mathrm{P}$ to get a certain temperature increase $\Delta \mathrm{T}$ of the waveguide core is: ${ }^{1}$

$$
P=\frac{2 \cdot T_{c} \cdot A \cdot \Delta T}{t}
$$

where $T_{c}$ is the thermal conductivity of the waveguide layer on the silicon substrate, $A$ is the heater area and $t$ is the thickness of the waveguide layer. It is assumed that the core temperature increase is about half the temperature increase of the heater. The heater area is about equal to the gap area and this is about the product of the required active section length $\mathrm{G}$ and twice the waveguide width, say $20 \mu \mathrm{m}$. The thickness of the waveguide layer is typically $30 \mu \mathrm{m}$. If the thermal conductivity of silica $\left(T_{c}=1.4 \mathrm{~W} \cdot \mathrm{m}^{-1} \cdot \mathrm{K}^{-1}\right)$ is used in the calculation, a worst-case value for $\mathrm{P}$ is found. This yields a power dissipation of only $6 \mathrm{~mW}$ for a phaseshift of $\pi$.

\section{POLYMER CHANNEL WAVEGUIDE SECTIONS IN SILICA WAVEGUIDES}

Hybrid polymer/silica waveguide devices based on polymer and silica channel waveguide sections are described in a patent by Kenney at al. ${ }^{6}$ Here we analyze a directional coupler switch as shown in fig. 2 . In the coupling region one of the two waveguide cores is made of polymer. A heater on top of the polymer waveguide activates the device. This induces a strong (effective) refractive index difference between the two waveguides due to the different sign of the thermo-optic coefficients of the two materials. A monolithic version, which depends on the weak slope of the temperature profile in the cross section of the coupling region, is less efficient due to the close proximity of the channels in that region. The required switch power for a single coupling length device can be estimated from the condition for reducing the coupling efficiency for the cross state from $100 \%$ to 0 . This requires a difference in effective indices $\Delta \mathrm{N}$ that satisfies the following equation: ${ }^{7}$

$\Delta N . L=\frac{\sqrt{3}}{2} \lambda$ 
where $\mathrm{L}$ is the coupling length.

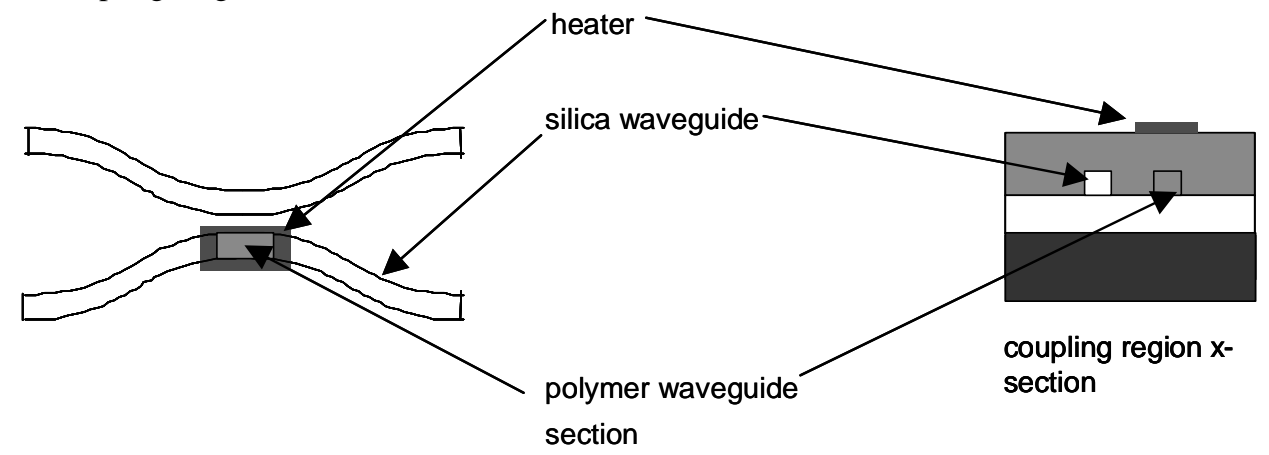

Fig. 3: Hybrid directional coupler switch using a polymer waveguide section.

The difference in effective indices can be approximated by the difference in the refractive indices: $\Delta \mathrm{N} \sim \Delta \mathrm{n}$. This in turn is about the change of the refractive index in the polymer only because of its much larger thermo-optic coefficient: $\Delta \mathrm{n} \sim\left(\mathrm{dn}_{\text {polymer }} / \mathrm{dT}\right) . \Delta \mathrm{T}$, where $\Delta \mathrm{T}$ is the temperature change of the polymer core required for switching.

$$
\frac{d n_{\text {polymer }}}{d t} \Delta T=\frac{\sqrt{3}}{2} \frac{\lambda}{L}
$$

The required refractive index change for the polymer is inversely proportional to the coupling length scaled to wavelength. Typical fiber compatible directional couplers have coupling lengths that range from $10 \mathrm{~mm}$ to $1 \mathrm{~mm}$. With such coupling lengths at a wavelength of $1.55 \mu \mathrm{m}$, the required index change in the polymer ranges from $1.5 \times 10^{-4}$ to $1.5 \times 10^{-3}$, which corresponds in glassy polymers to a core temperatures increase of only 1.5 to 15 degrees. A similar analysis of the required power dissipation as for the Mach Zehnder interferometer yields a value of about $14 \mathrm{~mW}$.

Hybrid polymer/silica integration is well suited for vertically stacked layer structures. After silica-on-silicon channel waveguide fabrication, polymer waveguide channels can easily be defined onto the silica top cladding. An elegant directional coupler switch structure as shown in fig. 4 was reported by Keil et al. ${ }^{8}$

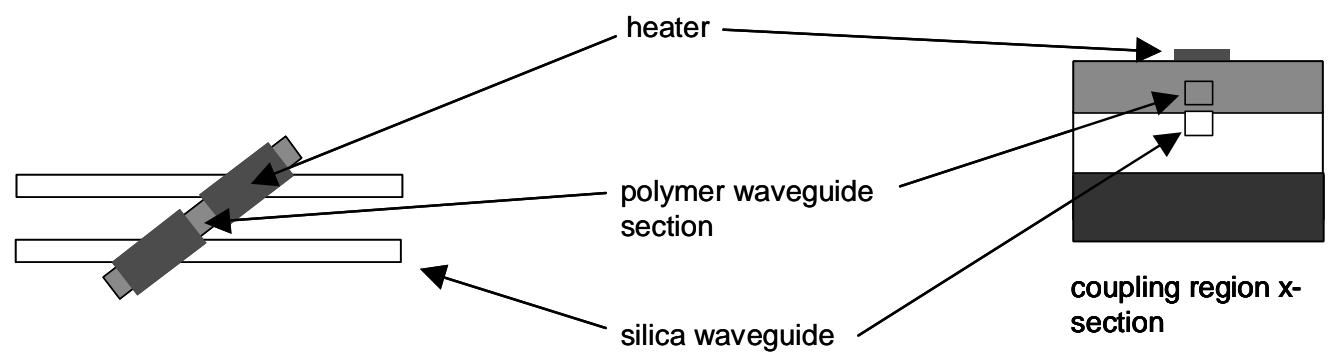

Fig. 4: Hybrid vertical directional coupler switch using a polymer waveguide section.

In their approach in-plane silica channels are coupled via short bridging polymer channels that lie on top of the silica channels. Tuning the effective index changes differentially by heaters on top of the polymer channels induces the vertical directional coupling between the channels. In fact it is a vertical version of the structure as shown in fig. 2 . 
The required switching power per coupler was $40 \mathrm{~mW}$ (two heaters have to be activated for switching). The insertion loss of a $1 \times 2$ switch was $1.5 \mathrm{~dB}$ and the crosstalk $-32 \mathrm{~dB}$.

\section{POLYMER CLADDINGS ON SILICA WAVEGUIDES}

Athermalization of Bragg gratings ${ }^{9}$, (micro ${ }^{10}$ )ringresonators ${ }^{11}$ and AWG's ${ }^{12}$ based on silica-on-silicon can be done by applying a polymer top cladding onto the silica channels. The low temperature processing and good planarization and gap filling properties of spin-coated polymers make this a convenient process. For these devices the condition for athermal behavior is:

$\frac{1}{\lambda_{0}} \frac{d \lambda_{0}}{d T}=\frac{1}{N} \frac{d N}{d T}+C T E=0$

where $\lambda_{0}$ is the Bragg, resonant or central wavelength, $\mathrm{N}$ is the effective index of the waveguide and $\mathrm{CTE}$ is the (linear) thermal expansion coefficient of the silicon substrate: $\mathrm{CTE}=2.6 \times 10^{-6}{ }^{\circ} \mathrm{C}^{-1}$.

This can be approximated by the following equation:

$\frac{1}{N}\left[\frac{d n_{\text {silica }}}{d T} \Gamma+\frac{d n_{\text {polymer }}}{d T}(1-\Gamma)\right]+C T E=0$,

where $\mathrm{dn}_{\text {silica }} / \mathrm{dT}$ is the thermo-optic coefficient of the silica core, $\mathrm{dn}_{\text {polymer }} / \mathrm{dT}$ is the thermo-optic coefficient of the polymer cladding and $\Gamma$ is the confinement factor of the mode. The latter depends on the refractive index difference between the core and the cladding. Therefore, in order to satisfy the condition for athermal behavior over a broad temperature range, the polymer cladding refractive index should not vary very rapidly. This favors the use of glassy polymers with relatively low thermo-optic coefficients. In that case a confinement factor of about 0.88 would be required. Such confinements can easily be created in fiber compatible single-mode waveguides by tuning the polymer cladding refractive index.

We have realized an athermal AWG using rubbery polymer claddings. ${ }^{13}$ Fig. 5 shows the temperature dependence of the fiber-compatible 16x16 AWG with $100 \mathrm{GHz}$ channel spacing.

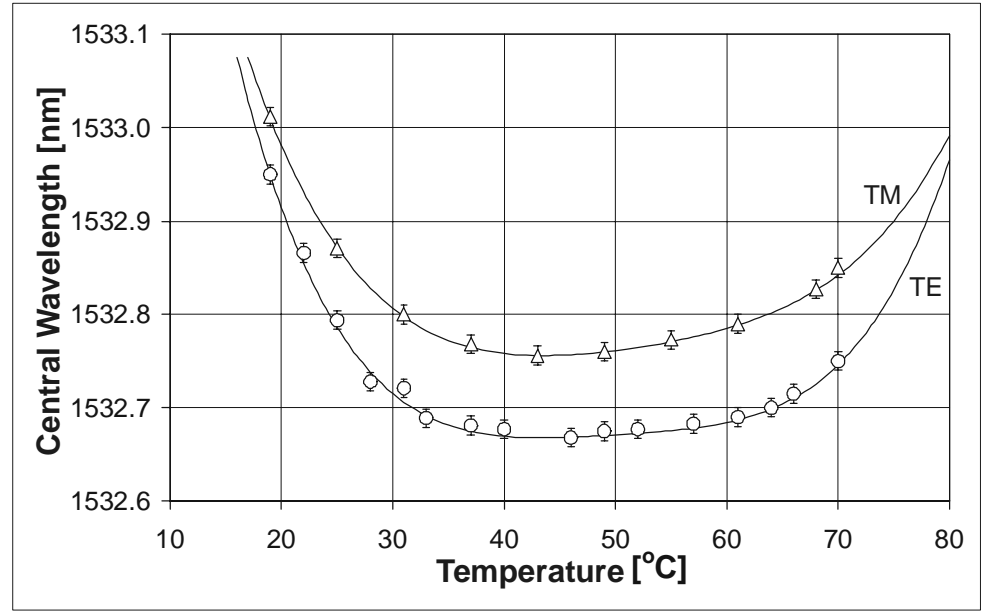

Fig. 5: Temperature and polarization dependence of the central wavelength of an AWG with a polymer cladding

The core is a $6 \times 6 \mu \mathrm{m}$ Ge-doped silica channel with $\mathrm{n}_{\text {silica }}=1.4534$. The bottom cladding is $16 \mu \mathrm{m}$ thick silica with $\mathrm{n}_{\text {silica }}=1.445$ and the rubbery polymer cladding is a $15 \mu \mathrm{m}$ thick layer with $\mathrm{n}_{\text {polymer }}=1.445 @ 20^{\circ} \mathrm{C}$ and $\mathrm{dn} \mathrm{n}_{\text {polymer }} / \mathrm{dT}=-$ 
$3.3 \times 10^{-4}$. All measurements are done at $1.55 \mu \mathrm{m}$. The temperature response is flat within $\pm 0.05 \mathrm{~nm}$ in the range between 20 and $70{ }^{\circ} \mathrm{C}$. The all-silica version has a shift that is of an order of magnitude larger.

From fig. 5 it can be concluded that the thermo-optic coefficient of the polymer cladding is too large. At the high temperature end, the polymer cladding refractive index has become so low, that the mode is confined almost completely in the core and this results in a temperature dependence that is determined only by the silica thermo-optic coefficient. At the low temperature end, the refractive index of the polymer cladding is approaching the silica core index and the mode starts to penetrate strongly in the polymer cladding. This results in a temperature dependence that is determined largely by the polymer thermo-optic coefficient.

An additional beneficial effect of the polymer cladding is the stress relieve of the core by the embedding in the low Young's modulus polymer cladding. This results in a vanishing birefringence in the waveguide core and consequently a reduced polarization dependence of the AWG response. The residual polarization dependence as shown in fig. 5, can be traced back to the birefringence $\left(3 \times 10^{-4}\right)$ in the polymer cladding.

Therefore, by applying a glassy, low birefringent polymer cladding we could reduce the temperature sensitivity and the polarization dependence of the AWG further than that what is shown in fig.5.

Polymer clad silica channels can be applied for switching applications also. We have demonstrated a non-planar waveguide switch based on fused fiber. ${ }^{14}$ The cross section of the device is shown in fig. 6 .

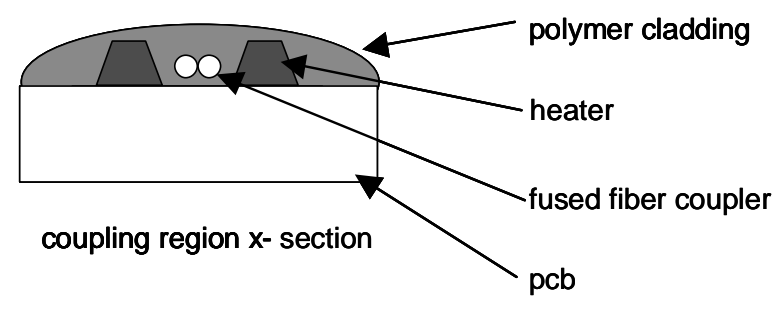

Fig. 6: Fused coupler switch using a polymer cladding

Weakly fused couplers having a dumbbell cross-section with fiber diameters reduced to $8 \mu \mathrm{m}$ were embedded in a silicone rubber with refractive index (@ $1.3 \mu \mathrm{m}$ ) $\mathrm{n}_{\text {polymer }}=1.3996$ which is of 0.5 lower than the fused fibers $\left(\mathrm{n}_{\text {silica }}=1.4467\right)$. The coupler was placed onto a printed circuit board containing copper heaters stripes to confine the coupling region $(5 \mathrm{~mm}$ long) of the coupler and to provide a way to change the refractive index of the polymer cladding. It was shown that the device could be switched completely at a power dissipation of about $1 \mathrm{~W}$. The insertion loss was less than $0.1 \mathrm{~dB}$. The coupling coefficient, $\mathrm{C}$, of a weakly fused coupler can be described by the following equation: ${ }^{15}$

$$
C=\frac{2.405^{2}}{\pi^{3}} \frac{\lambda^{5 / 2}}{a^{7 / 2}\left(n^{2}{ }_{\text {silica }}-n^{2}{ }_{\text {polymer }}\right)^{2} \cdot n_{\text {silica }}}
$$

where $\mathrm{a}$ is the core diameter.

The condition for switching is:

$\Delta C . L=\pi$

where $\Delta \mathrm{C}$ is the thermo-optically induced change in the coupling coefficient due to the change in the refractive index of the polymer cladding and, to a lesser degree, the silica core. For the devices as realized, the equation predicts a required increase of the cladding temperature of only a few degrees to induce switching. The large distance between 
the heaters and the cores and the heat leakage via the thick copper heaters explain the relatively high power dissipation that was found experimentally. A simple way to improve this can be derived from the equation for the coupling constant. By tuning the polymer index closer to the silica index, a large reduction in power dissipation can be obtained. The distance between the core and the heaters and the heat leakage via the heaters can be reduced easily when a planar waveguide technology is applied as shown in fig. 7.

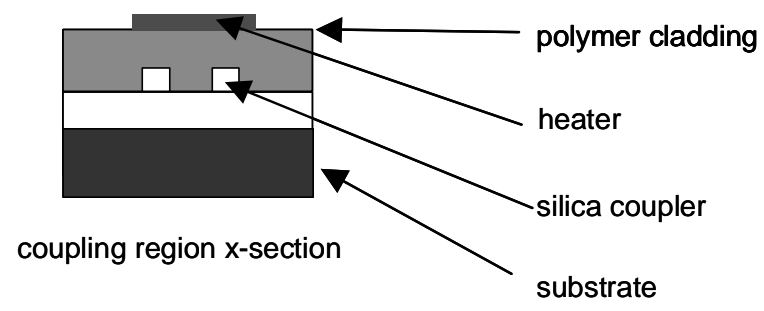

Fig. 7: Hybrid directional coupler switch using a polymer cladding

Such a device is claimed in a European patent by Bosc at. al. ${ }^{16}$

A simple-to-realize thermo-optic attenuator based on a silica core with a polymer cladding can be obtained by stretching an optical fiber in a flame such that the diameter is reduced to several microns. By applying a polymer cladding with a higher refractive index onto the stretched section, the resultant waveguide will become leaky. Fig. 8 shows the cross section of such a device. However by increasing the temperature of the cladding such that the refractive index of the polymer cladding drops below the silica core, the structure becomes a low-loss waveguide. This temperature increase could be induced by providing the substrate with heaters. Fig. 9 shows the temperature dependence of the insertion loss of such a stretched fiber (@ $1.55 \mu \mathrm{m}$ ) embedded in a silicone polymer cladding with $\mathrm{dn}_{\text {polymer }} / \mathrm{dT}=-3.3 \times 10^{-4}$. It can be seen that around $125^{\circ} \mathrm{C}$ the insertion loss can be changed from $-40 \mathrm{~dB}$ to less than $2 \mathrm{~dB}$ in a temperature range of only 10 degrees. The transition temperature can be selected by tuning the polymer refractive index. A drawback of this device is the presence of oscillations in the attenuation characteristic. This makes the device more suitable for shutter applications than for variable attenuator applications. The origin of these oscillations lies in the directional coupling between the coaxial rod and the tube waveguides formed by the fiber core and cladding in the transition region between the original fiber and the stretched fiber. ${ }^{17}$

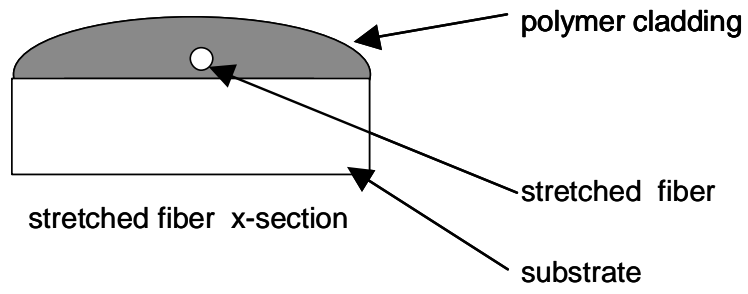

Fig. 8: Stretched fiber attenuator using a polymer cladding 


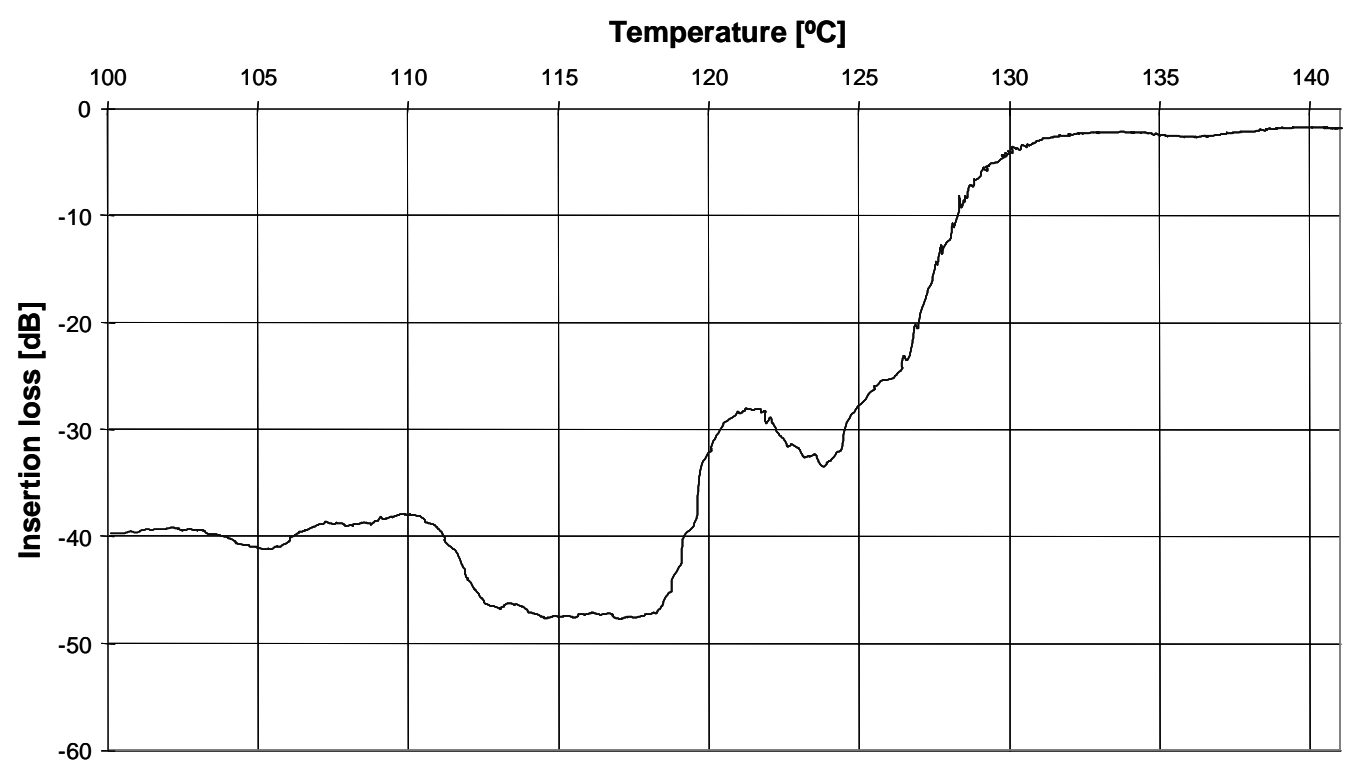

Fig. 9: Temperature dependence of the insertion loss of a stretched fiber with polymer cladding

A planar waveguide version of the device described above consisting of a silica-on-silicon waveguide having a core that is locally provided with a polymer cladding is presented in ${ }^{18}$. Its schematically structure is shown in fig. 10 . This device has a smooth attenuation characteristic that ranges from $-22 \mathrm{~dB}$ at low temperatures to $2.5 \mathrm{~dB}$ at high temperatures.

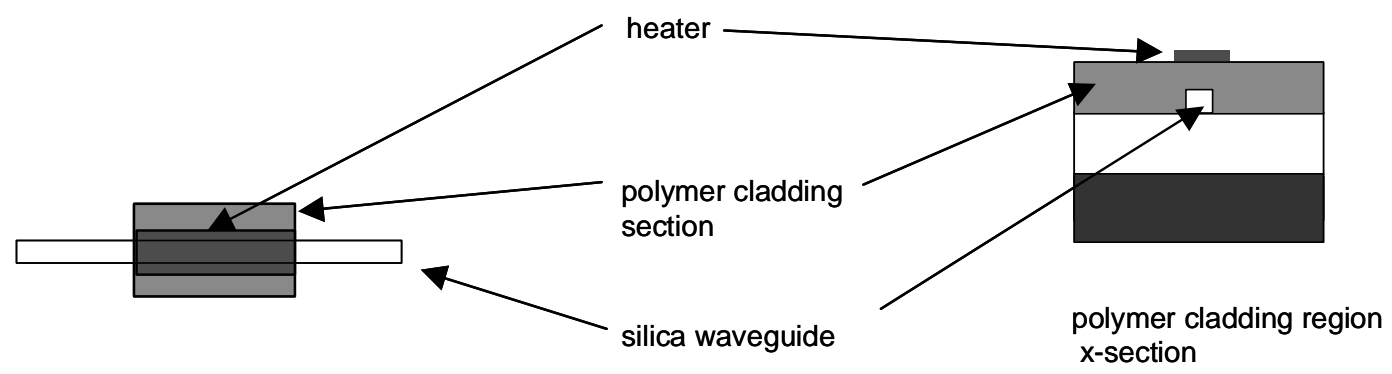

Fig. 10: Hybrid straight waveguide variable optical attenuator using a polymer cladding

The excess loss of a bent waveguide has a strong dependence on refractive index contrast below a certain critical value of this contrast. ${ }^{19}$ This effect can be utilized in polymer clad bent silica waveguides or polymer channel waveguides embedded in silica to create efficient thermo-optic variable attenuators or shutters. ${ }^{20}$ A schematically structure is shown in fig. 11. The polymer cladding has a small refractive index difference with the core in absence of thermal activation. The device is designed to be in the high loss state then. Upon activation by the heater, the contrast increases and the bend loss drops to a low value. 


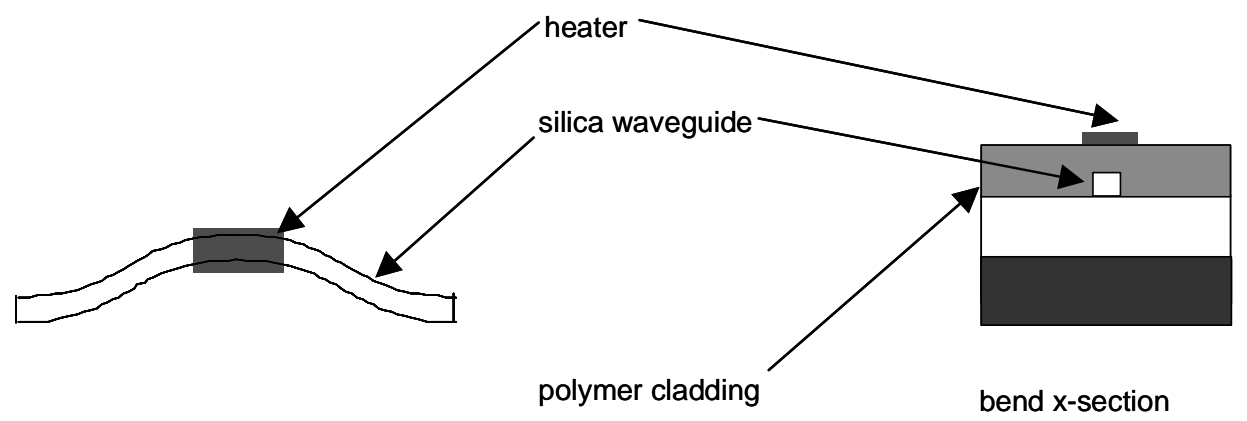

Fig. 11: Hybrid bent waveguide variable optical attenuator using a silica core and a polymer cladding

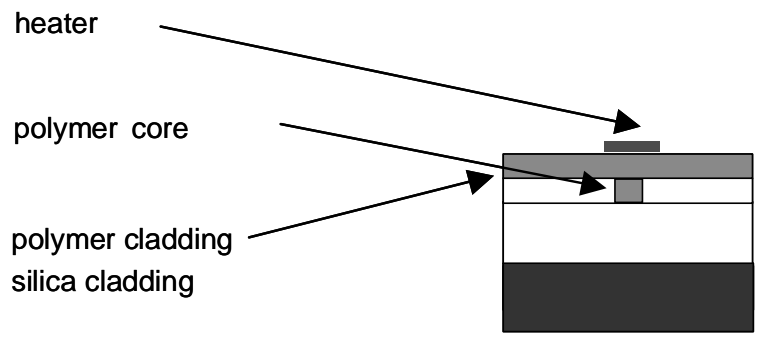

bend $\mathrm{x}$-section

Fig. 12: Hybrid bent waveguide variable optical attenuator using a polymer core and a silica/polymer cladding

The alternative structure as shown in fig. 12 switches from the low loss state to the high loss state upon activation of the heater. The hybrid bent channel attenuator has been analyzed and realized in the structure as shown in fig. 12 using a siliconoxynitride technology. ${ }^{21}$ For fiber compatible channels $(5 \times 5 \mu \mathrm{m}$, contrast 0.006$)$ the theoretically required refractive index change of the polymer to obtain $30 \mathrm{~dB}$ attenuation is about $10^{-3}$ for a device length of $9 \mathrm{~mm}$. This index change corresponds to a (glassy) polymer temperature increase of only 10 degrees. The experimental device required more than $1 \mathrm{~W}$ to attenuate by $30 \mathrm{~dB}$. This high value can be explained by refractive index lowering of polymer top cladding counteracting the loss of effective index contrast. In addition, the polymer top cladding acts a thermal insulator for the core. There will be ea relatively large temperature drop across the top cladding due to its low thermal conductivity compared that of to silica. Finally, the polymer core thermal expansion and the refractive index change associated with that are restricted by the stiff silica cavity walls. It is therefore expected that the structure of fig. 11 would behave more efficiently.

\section{CONCLUSIONS}

In this paper we have presented a number of hybrid optical waveguide devices based on polymers and silica. It is shown that the excellent thermo-optic properties of polymers can be combined with the superior waveguiding properties of silica resulting in devices with enhanced performance. In addition it is shown that the hybrid integration can take advantage of the largely different thermo-optic properties of both classes of materials to make devices that are either highly temperature sensitive or insensitive (athermal).

It must be mentioned however, that all switches and attenuators described in this paper, with the exception of the hybrid Mach Zehnder interferometer, are sensitive for ambient temperature changes. Therefore, temperature 
stabilization would be required to allow for practical applications. That limits their application to high-end applications or to ambient temperature tolerant applications, such as shuttering or variable attenuation with closed-loop control.

\section{REFERENCES}

1. M. Diemeer, P. De Dobbelaere and R. Flipse, Integrated Optical Circuits and Components, E.J. Murphy ed., Chap. 8, Marcel Dekker, N.Y., 1999

2. K. Okamoto, Integrated Optical Circuits and Components, E.J. Murphy ed., Chap. 4, Marcel Dekker, N.Y., 1999

3. Y. Inoue, A. Kaneko, F. Hanawa, H. Takahashi, K. Hattori and S. Sumida, “ Athermal silica-based arrayedwaveguide multiplexer," Electron. Lett., 33, (23), pp. 1945-1947, 1997

4. D. Marcuse, "Loss analysis of single-mode fiber splices," B.S.T.J., 56, (5), pp. 703-718, 1977

5. A. Kaneko, S. Kamei, Y. Inoue, H. Takahasi and A. Sugita, " Athermal silica-based arrayed-waveguide grating (AWG) multiplexers with new low loss groove design," OFC'99, Proc., TuO1-1, pp. 204-206, 1999

6. J.T. Kenney, J. Midgley, V.N. Morozov, K. Purchase, M. Stiller, A. Thicknor, J.Burke and J. Love, “ Photonic devices comprising thermo-optic polymer," United States Patent, US 6,311,004 B1, Oct. 30, 2001

7. R.C. Alferness, Guided-wave optoelectronics, T. Tamir ed., Chap. 4, Springer-Verlag, N.Y., Berlin, Heidelberg, 2-ed, 1990

8. N. Keil, H.H. Yao, C. Zadwadzki, K. Lösch, K. Satzke, W. Wischmann, J. v. Wirth, J. Schneider, J. Bauer and M. Bauer, "Hybrid polymer/silica vertical coupler switch with $<-32 \mathrm{~dB}$ polarization-independent crosstalk," Electron. Lett., 37, (2), pp. 89-90, 2001

9. D. Bosc, B. Loisel, M. Moisan, N. Devoldere, F. Le Gall and A. Roland, " Temperature and polarization insensitive Bragg grating realized on silica on silicon," Electron. Lett., 33, (2), pp. 134-136, 1997

10. S. T. Chu, W. Pan, S. Suzuki, B. Little, S. Sato and Y. Kokoburn, " Temperature insensitive vertically coupled microring resonator add/drop filters by means of a polymer overlay," IEEE Photon. Technol. Lett., 11, (9), pp. 1138-1140, 1999

11. Y. Kokoburn, S. Yoneda and H. Tanaka," Temperature-independent narrow-band filter by athermal waveguide," ECOC'96, Proc.,WeD.1.5, pp. 3.143-3.146, 1996

12. M.B.J. Diemeer and T.H. Hoekstra, " Phased array wavelength multiplexer," International Patent Application, WO 99/21038, 1999

13. J. van Nunen, "Realization of an arrayed waveguide grating with low polarization- and low temperature dependence," Master Thesis, University of Twente, Lightwave Devices Group, Sept. 2000

14. M.B.J. Diemeer, W.J. de Vries and K.W. Benoist, "Fused coupler switch using a thermo-optic cladding," Electron. Lett., 24, (8), pp. 457-458, 1988

15. F.P. Payne, C.D. Hussey and M.S. Yataki, "Polarization analysis of strongly fused and weakly fused tapered couplers", Electron. Lett., 21, (13), pp. 561-563, 1985

16. D. Bosc and N. Devoldere, "Coupleur directif mixte silice/polymère en optique intégrée," European Patent, EP 0797113 A1, 1997

17. A.C. Boucouvalas and G. Georgiou, "External refractive index response of tapered coaxial couplers," Electron. Lett., 11, (4), pp. 257-259, 1986

18. T. Ticknor, "Integration of multifunctional planar waveguide circuits", Lightwave, 18, (3), p. 224, 2001

19. F. Ladouceur and J.D. Love, Silica-based buried channel waveguide and devices, Chapmann \& Hall, London, 1996

20. G.J. Veldhuis, P.V. Lambeck, M.B.J. Diemeer and T.H. Hoekstra, “ Optical intensity modulator and switch comprising the same," United States Patent, US 6,377,716, 2002

21. G.J. Veldhuis, F.N. Krommedijk and P.V. Lambeck, "Integrated optic modulator based on a bent channel waveguide", Opt. Comm ., 168, pp. 481-491, 1999 